\title{
EDITORIAL
}

\section{The drugs can work}

\author{
There are signs that the therapeutic successes that have contributed to the downward \\ trajectory for HIV-1 mortality may soon be repeated for HCV.
}

A recent paper in the Annals of Internal Medicine revealed a statistic that at first glance may seem startling: hepatitis $\mathrm{C}$ virus (HCV) now causes more deaths per year in the United States than HIV-1 (REF. 1). In a survey of death certificate data, Ly et al. found that in 2007 at least 15,000 deaths were directly related to HCV infection, whereas HIV-1 infection accounted for just over 12,000 deaths. An estimated 3.2 million individuals in the United States are living with chronic HCV infection, and there are thought to be $\sim 30,000$ new infections each year. By contrast, an estimated 1.2 million people are living with HIV-1 infection, and there are $\sim 50,000$ new infections each year. The downward trend in HIV-related mortality is being reflected in many other countries as access to antiretroviral therapy (ART) increases. The 2011 WHO report on the global HIV/AIDS response indicated that there has been a $22 \%$ decline in AIDS-related mortality worldwide over the past 5 years ${ }^{2}$.

In the 30 years that have passed since the first report of an AIDS-like illness, there has been an amazing amount of work on the molecular, cellular and structural biology of HIV-1 and the response of the host to HIV-1 infection. The first antiretroviral drug, azidothymidine (AZT), was approved for use in 1987, just 3 years after HIV-1 was identified. Since then, ART has grown more and more sophisticated, and many of the current highly active ART (HAART) regimens are now well tolerated and highly effective, with the best combinations able to reduce the amount of HIV-1 RNA in plasma to undetectable levels. In fact, although there is still work to be done to improve HAART, over the past 18 months the discussion around ART has taken an unexpected turn, with commentators now talking about effective control of HIV/AIDS through a combination of treatment-as-prevention strategies (treating HIV-positive individuals with ART in order to prevent transmission) and drug- or microbicide-based preexposure prophylaxis (PrEP) strategies. Although there are still many challenges ahead, including the need to scale up diagnosis and monitoring, as well as a raft of social and ethical issues to discuss, not to mention the fiscal implications of such strategies, it seems that control of the disease - even in the absence of a vaccine - may be possible in the future.
What about HCV? Approximately 170 million individuals worldwide, or $\sim 3 \%$ of the global population, are infected. The virus, a positive-sense RNA virus belonging to the Flaviviridae, establishes a chronic infection in the liver that persists for decades and eventually causes diseases such as cirrhosis and hepatocellular carcinoma. Despite the enormous burden of infection, HCV remains low profile in terms of public awareness. This is likely to change in the near future, however, as the current majority of individuals with $\mathrm{HCV}$ infections were born between 1945 and 1964 (the so-called baby boomer generation) and are therefore now reaching the age at which HCV-related diseases become apparent.

For more than 10 years the gold standard HCV therapy has been weekly injections of pegylated interferon (PEG-IFN) combined with a daily oral dose of the broad spectrum antiviral ribavirin. The treatment course lasts almost 1 year, achieves a sustained virological response (SVR) in only $50 \%$ of patients and can have severe adverse effects. Patient uptake and compliance rates are therefore very poor. The treatment spectrum changed dramatically last year, however, when two NS3-4A protease inhibitors, telaprevir (from Vertex) and boceprevir (from Merck), the first direct-acting antivirals (DAAs) for $\mathrm{HCV}$, were approved for use in Europe and the United States. Used in combination with (PEG-IFN)ribavirin, the protease inhibitors substantially boost the achievable SVR in patients infected with HCV genotype 1 and can greatly reduce the length of treatment for responsive patients; however, they also enhance the adverse effects associated with the treatment. The focus for second- and third-generation DAAs is therefore on the development of an oral, IFN-free combination therapy, and early results of combinations involving protease and polymerase inhibitors look promising.

Of course, for HIV/AIDS the 'holy grail' remains a vaccine, and intensive vaccine research is also ongoing for $\mathrm{HCV}$, although the genetic diversity of this virus poses a particular challenge. In the meantime, however, it seems that the drugs can work. It is therefore vital to ensure that all those who are eligible for drug treatment can gain access.

\footnotetext{
Ly, K. N. et al. Ann. Intern. Med. 156, 271-278 (2012).

2. WHO. Progress report 2011: global HIV/AIDS response. WHO [online], http://www.who.int/hiv/pub/progress_report2011/en/index.html (2011).
} 